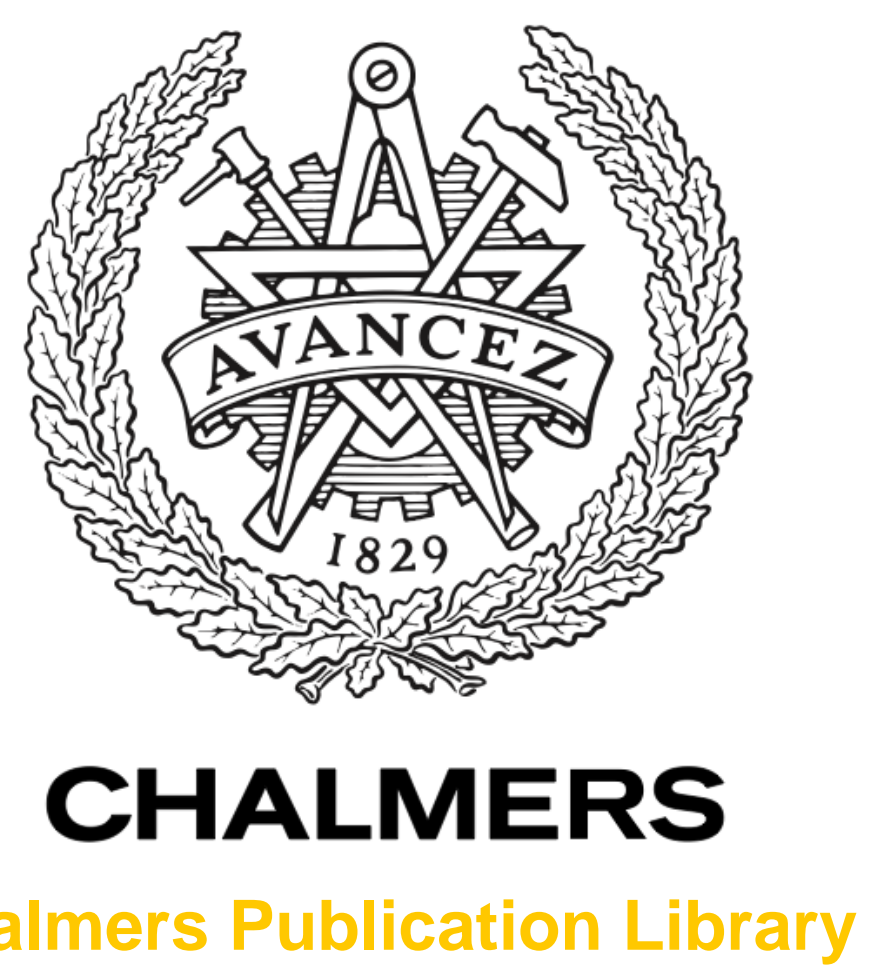

\title{
Measurements of RFID Tag Sensitivity in Reverberation Chamber
}

This document has been downloaded from Chalmers Publication Library (CPL). It is the author's version of a work that was accepted for publication in:

IEEE Antennas and Wireless Propagation Letters (ISSN: 1536-1225)

Citation for the published paper:

Hidén Rudander, J. ; Ikram E-Khuda, I. ; Kildal, P. (2011) "Measurements of RFID Tag Sensitivity in Reverberation Chamber". IEEE Antennas and Wireless Propagation Letters, vol. 10 pp. 1345 â 1348.

http://dx.doi.org/10.1109/LAWP.2011.2177512

Downloaded from: http://publications.lib.chalmers.se/publication/153317

Notice: Changes introduced as a result of publishing processes such as copy-editing and formatting may not be reflected in this document. For a definitive version of this work, please refer to the published source. Please note that access to the published version might require a subscription.

Chalmers Publication Library (CPL) offers the possibility of retrieving research publications produced at Chalmers University of Technology. It covers all types of publications: articles, dissertations, licentiate theses, masters theses, conference papers, reports etc. Since 2006 it is the official tool for Chalmers official publication statistics. To ensure that Chalmers research results are disseminated as widely as possible, an Open Access Policy has been adopted.

The CPL service is administrated and maintained by Chalmers Library. 


\title{
Measurements of RFID Tag Sensitivity in Reverberation Chamber
}

\author{
Jacob Hidén Rudander, Ikram-e-Khuda, Per-Simon Kildal, Fellow, IEEE, and Charlie Orlenius
}

\begin{abstract}
The reverberation chamber is now commonly accepted and used for fast and accurate performance evaluation of mobile phones and other wireless devices for in particular new systems such as LTE 4G and IEEE 802.11n Wi-Fi. In this letter, we describe a new methodology of measuring sensitivity of RFID tags in a reverberation chamber, and we show how the measurements time can be shortened by using the fact that the RFID tag behaves like an ideal threshold receiver. We then extend to tag population measurements that can be used to select the best tag among the many during the same measurement sequence.
\end{abstract}

Index Terms-Anechoic chamber, fading, Rayleigh fading, reverberation chamber, RFID.

\section{INTRODUCTION}

$\mathbf{N}$ OWADAYS, UHF RFID tags can be seen in a diverse area of applications ranging from tracking and identification of goods in retail and logistic applications to on-the-fly identification of railway wagons [1]. In many of the applications where RFID is used, the maximum reading range is one of the most important design parameters to ensure good functionality [2]. In a typical passive RFID system, the maximum reading range is limited by the sensitivity of the tags and not by the reader [3]. Hence, ways to measure tag sensitivity are of great importance.

The standardized way to measure sensitivity of passive UHF RFID tags is in anechoic chambers, corresponding to location of the RFID in free space. However, RFID tags will never be located in free space, and therefore the characterization in anechoic chambers is an inherited tradition and not optimum from the technical point of view. This is well known [2], [4], and methods to compensate results measured in free space for reallife locations are being developed. It is much more natural to characterize RFID tags in complex environments with a lot of multipath. This letter deals with characterizing passive UHF

This work was supported in part by the Swedish

Transport Administration and the Swedish Governmental Agency for Innovation Systems (VINNOVA) within the VINN Excellence Center Chase.

J. H. Rudander was with Chalmers University of Technology, Gothenburg

43543, Sweden. He is now with Kongsberg Maritime, Horten 3183, Norway

(e-mail: jacob.hiden.rudander@kongsberg.com).

Ikram-e-Khuda was with Chalmers University of Technology, Gothenburg 43543, Sweden.

P.-S. Kildal is with Chalmers University of Technology, Gothenburg 43543, Sweden (e-mail: per-simon.kildal@chalmers.se).

C. Orlenius is with Bluetest AB, Gothenburg 41755, Sweden.
RFID tags in such a well-defined statistically isotropic multipath environment, the reverberation chamber [5].

The reverberation chamber, or mode-stirred chamber, is a metal cavity equipped with mode stirrers. The cavity is large enough to support several resonant modes at a frequency band of interest [6], with each mode representing eight plane waves. The modes are excited by a transmitting antenna and stirred by different techniques: movement of mechanical structures, movement of device under test (DUT), referred to as platform stirring, or switching between different transmitting antennas, referred to as polarization stirring. After the year 2000, the reverberation chamber has been developed for accurate measurements of antenna efficiency, diversity gain, and capacity of multiple-input-multiple-output (MIMO) antenna systems [7], radiated power of active devices like mobile phones, receiver sensitivity [8], and lately also throughput data rate of wireless devices for LTE 4G [9] and IEEE 802.11n Wi-Fi systems.

The theory of the reverberation chamber is well known [10], as well as the measurement uncertainty by classical complex Gaussian theory [11]. The uncertainty has been evaluated by many round-robin tests for active devices, and in particular by comparing results for efficiency, correlation, diversity gain, and maximum available MIMO capacity obtained from passive measurements in an anechoic chamber and reverberation chamber; see, e.g., [12].

When measuring active devices, it is also important to know and control the time delay spread and coherence bandwidth in the measurement environment to resemble real-life situations. This is easily done in the reverberation chamber [13]-[15].

\section{RFID SENSITIVITY MEASUREMENTS}

The RFID tag sensitivity in this letter was carried out in the anechoic chamber at Chalmers University of Technology and in the Bluetest HP reverberation chamber, both in Gothenburg, Sweden, by means of a monostatic RFID reader (Scirocco R600) using frequency-hopping spread spectrum (FHSS) between four channels $(865.7,866.3,866.9$, and $867.5 \mathrm{MHz})$ in the UHF range. The Scirocco RFID reader had the capability to read 170 96-bit electronic product code (EPC) values per second. The calculation of read 96-b EPCs in percentage, mentioned several instances in this letter, was done after completed measurement by calculating the fraction of the actual number of read 96-bit EPC values reported back to the controlling software during the measurement and the theoretic maximum of read 96-bit EPC values during the time for the measurement. The sensitivity of the Scirocco RFID reader was unknown, but this is considered to be of less importance since this letter examines relative differences between results measured in the anechoic chamber and reverberation chamber. 


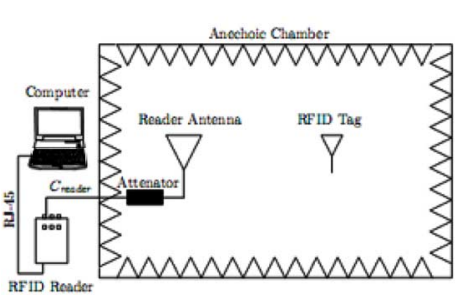

(a)

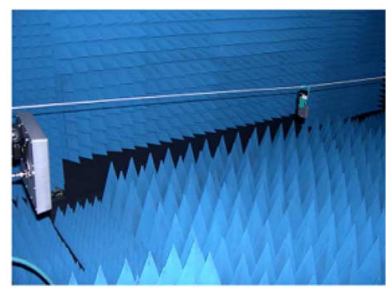

(b)
Fig. 1. (a) Drawing and (b) photograph of the measurement setup in anechoic chamber for tag sensitivity measurements.

The tags used were passive tags following the GS1 EPC global Class-1 Generation-2 UHF RFID standard, and each tag carried a 96-bit EPC value. Tags optimized for different environments and manufactured by different vendors were used to represent tags of different quality.

\section{A. In Anechoic Chamber}

The tags were measured one at the time, located at a distance of $1 \mathrm{~m}$ from the reader antenna, a circular-polarized 8-dBi UHF antenna. The tag was located in such a way that its front side, where the antenna is mounted, was facing the reader antenna, as shown in Fig. 1.

The power transmitted from the reader was gradually decreased in steps of $1 \mathrm{~dB}$, using a programmable attenuator. The lowest reader power $P_{\text {reader }}$ was taken as the mean value of the last power level when the tag was able to respond with at least 50\% 96-bit EPCs, and the first power level when the tags were not able to respond with 50\% 96-bit EPCs, during a 20 -s-long measurements. The sensitivity threshold in the tags' main beam was then found from the Friis transmission formula

$$
P_{\mathrm{th}, \mathrm{AC}}=\left(\frac{\lambda}{4 \pi r}\right)^{2} G_{\text {reader }} X L_{c} L_{a} P_{\text {reader }}
$$

where $X$ is the polarization mismatch, and $L_{c}$ and $L_{a}$ are transmission factors accounting for losses in cables.

\section{B. In Reverberation Chamber}

The measurement setup is shown in Fig. 2 with the tag under test separated by at least a half-wavelength from any metal object in the chamber. The RFID reader was connected via an attenuator to the reverberation chamber's wall-antennas. The reverberation chamber was loaded with a head phantom to increase the coherence bandwidth of the chamber [13] in order to minimize EPC errors as an effect of a channel that decorrelates over frequency, i.e., we wanted to ensure frequency-flat fading.

Measurements were made using one tag at the time. The stirrers were moved continuously for $20 \mathrm{~s}$ while the number of 96-bit EPC reads was counted. The average transmitted power into the chamber was decreased in steps of $1 \mathrm{~dB}$ using a programmable attenuator, and the procedure was repeated. The lowest reader power $P_{\text {reader }}$ was taken as the mean value of the last power level when the tag was able to respond with at least 50\% 96-bit EPCs, and the first power level when the tag was not able to respond with 50\% 96-bit EPCs. In this way, the average fading sensitivity (AFS) [8] of the tag at 50\% EPC level was

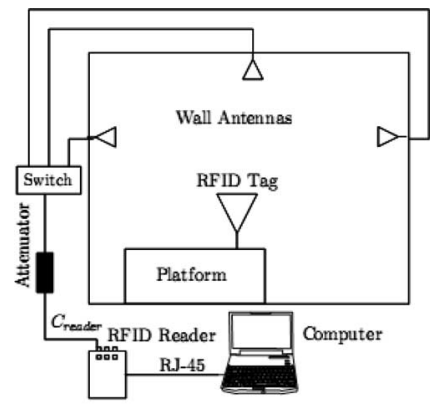

(a)

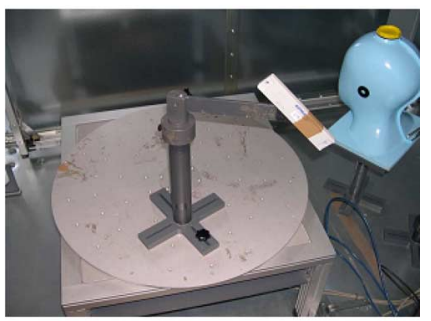

(b)
Fig. 2. (a) Drawing and (b) photograph of the measurement setup in reverberation chamber for tag sensitivity measurements.

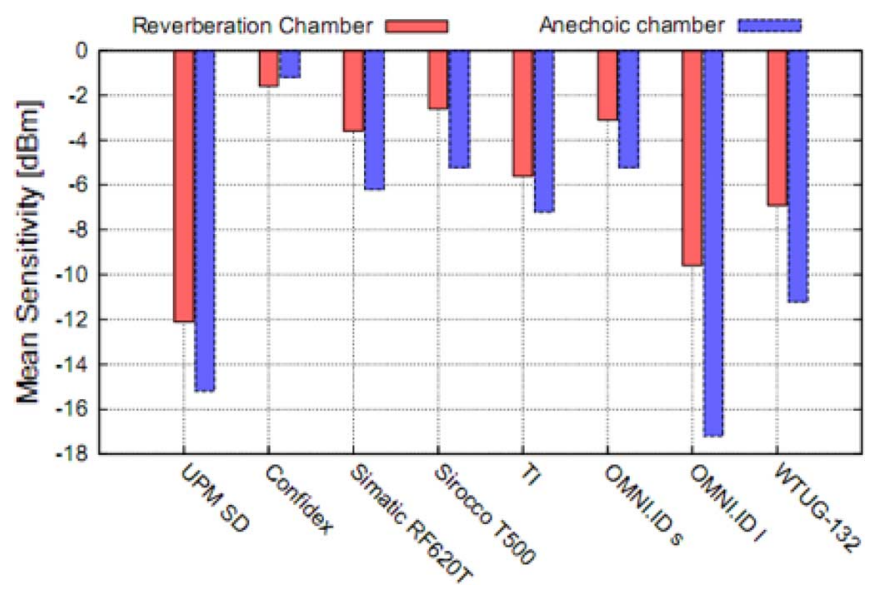

Fig. 3. Measured relative tag sensitivity in reverberation chamber and anechoic chamber using a normalized reader power.

measured, and the corresponding sensitivity threshold was then calculated from the chamber reference function $G_{\text {ref }}$ by using

$$
P_{\mathrm{th}, \mathrm{RC}}=G_{\mathrm{ref}} L_{c} L_{a} P_{\text {reader }}
$$

where $L_{c}$ and $L_{a}$ are the same as in (1). The chamber reference function is obtained from the calibration of the chamber and represents the average power transfer function.

\section{Results and Discussion}

The sensitivity of different RFID tags was measured in both anechoic and reverberation chambers as explained above. The results can be seen in Fig. 3.

For the reverberation chamber, the measurements were repeated five times by locating the tag in five different positions and orientations, whereas this did not make sense in the anechoic chamber because then the tag needs to point toward the reader. The standard error around the average for each tag was found to be $0.48 \mathrm{~dB}$, which is in agreement with the stated uncertainty of $0.5 \mathrm{~dB}$ of the reverberation chamber. We see from Fig. 3 that if we range the tags according to their sensitivity, the order will be the same for both measurement methods (except one case, "OMNI.ID large," due to its high directivity), hence we can conclude that AFS sensitivity measurements in reverberation chambers actually make sense and are a parameter characterizing the performance of the tags. We further believe the AFS value will be a more generally valid design parameter for RFID 
systems than sensitivity under line of sight (LOS) since there always will be some multipath effects present in real environments that cause an unpredictable performance unless averaging is done over many different realizations like in the reverberation chamber.

There is a systematic difference between the sensitivities measured in anechoic and reverberation chambers. The reason is that the sensitivity in the anechoic chamber is proportional to the realized gain (i.e., total radiation efficiency times directivity) of the tag antenna in the direction of the RFID reader, and in the reverberation chamber case, it is only proportional to the total radiation efficiency of the tag antenna (i.e., product of ohmic losses and mismatch factor) [5]. The directivity plays no role anymore.

The impact of the directivity can clearly be seen as the large difference between the sensitivity results in anechoic and reverberation chambers for the "OMNI.ID large" tag. The "OMNI.ID large" tag was, compared to the other tags, a huge tag with high directivity designed to work at distances up to $35 \mathrm{~m}$; i.e., it was designed for use in free-space-like environments with a dominant LOS. This is not a common location for RFID tags.

It should also be noted that the Confidex tag showed higher sensitivity in the reverberation chamber than in the anechoic chamber. We believe the reason for this is that the Confidex tag is designed for being located on a metal plate, whereas this was not the case in our measurements. Therefore, the radiation pattern may not be as directive as it should be, and the main lobe may not even be pointing toward the reader. This will affect the measured sensitivity in the anechoic chamber, but not on the reverberation chamber that is an isotropic environment, where orientation and directivity does not matter, hence giving the higher sensitivity there.

\section{Fast Estimation From Single-Power-LeVel MEASUREMENT IN REVERBERATION CHAMBER}

The tag can be regarded as an ideal threshold receiver as defined in [9] because the EPC reads will be reported at "every" instance when the power available to the tag is above the threshold $P_{\mathrm{th}}$, and there is nothing reported below. The reverberation chamber has Rayleigh fading, so the received power samples are exponentially distributed, making the relative number of reads (corresponding to the throughput in [9]) satisfying

$$
\operatorname{Read}_{\%}(\bar{P})=100 \% \cdot \operatorname{CCDF}\left(P_{\text {th }} / \bar{P}\right)=100 \% \cdot e^{-P_{\text {th }} / \bar{P}}
$$

where $\bar{P}$ is the average power incident on the tag, being corrected for the average power transfer function of the chamber, and CCDF is the complementary cumulative power distribution of the power samples at the threshold level.

The relation in (3) makes it possible to estimate the sensitivity of the tag already after measuring with one average power level, i.e., we measure the percentage of number of successful 96-bit EPC reads at one transmitted power level, correct the transmitted power level with the average power transfer function of the chamber to get $\bar{P}$, and determine $P_{\mathrm{th}}$ from (3). This is much faster than searching for the 50\% successfully read 96 -bit EPCs as explained in Section II.

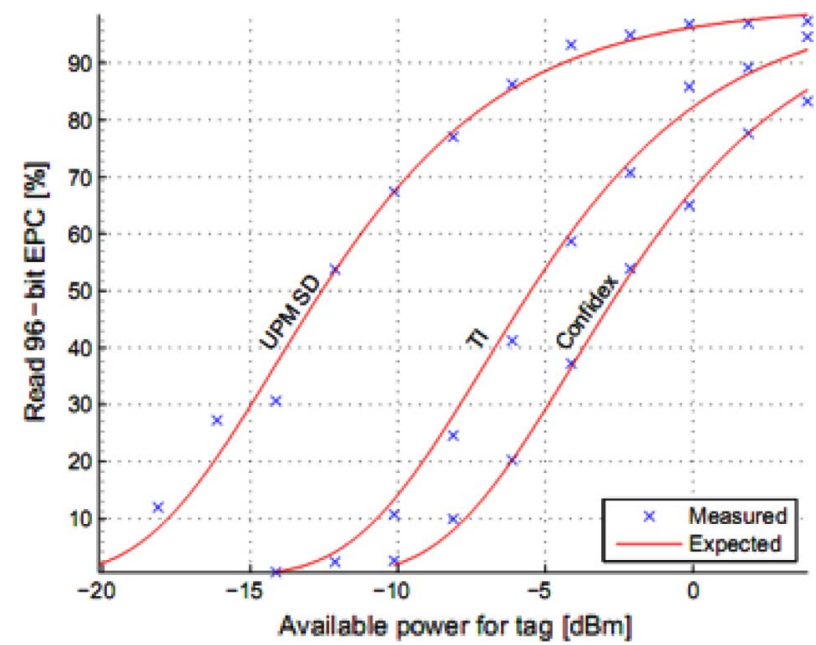

Fig. 4. Measured 96-bit EPCs and expected tag AFS sensitivity in reverberation chamber

TABLE I

Standard Deviation of $P_{\text {th }}$ Using Single Power Point Estimations From Measured Percentages and Different Average CHANNEL POWERS

\begin{tabular}{|l||c||c|}
\hline Tag Name & STD $[\mathrm{dB}]$ & $\begin{array}{c}\text { Number of } \\
\text { Estimations }\end{array}$ \\
\hline UPM SD & 1 & 13 \\
Confidex & 0.33 & 8 \\
OMNI.ID large & 1.3 & 10 \\
OMNI.ID small & 0.3 & 11 \\
Simatic RF620T & 0.6 & 10 \\
Scirocco T500 & 0.5 & 13 \\
TI & 0.4 & 8 \\
WTUG-132 & 0.5 & 14 \\
\hline
\end{tabular}

We see in Fig. 4 the number of read 96-bit EPCs in percentage for different average power levels together with a theoretically "expected" CCDF curve. We have produced the CCDF curve by first estimating $P_{\text {th }}$ from each average power level and its 96-bit EPC value using (3), then averaging these $P_{\text {th }}$ estimates, and finally producing an "expected" CCDF curve using (3) with the averaged $P_{\mathrm{th}}$.

We see in Fig. 4 that the expected CCDF curve with an ideal threshold receiver corresponds very well with the measured values. This proves that the ideal threshold receiver with threshold $P_{\mathrm{th}}$ is a good approximation to the tags real behavior. This fact can be used to speed up sensitivity measurements in reverberation chambers since it is possible to estimate a tags sensitivity threshold $P_{\text {th }}$ with good accuracy from just a few measurements made at a fairly arbitrary average channel power. This was verified by repeated single-power-level measurements. The standard deviations of the results are shown in Table I for the different tags. The results are good except for the directive "OMNI.ID large" tag.

\section{Tag Population Measurements}

The purpose of tag population measurements is to determine the relative quality between tags in a population of tags. The basic idea is to rank the tags by examining their number of responses since more sensitive tags are likely to respond more times in the fading reverberation chamber environment. 


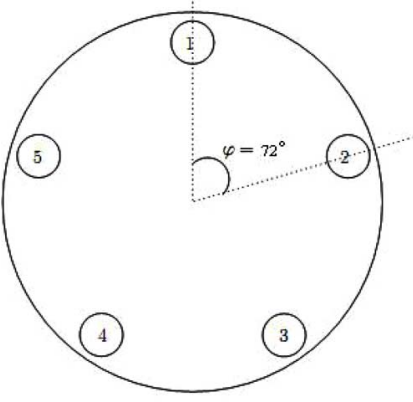

(a)

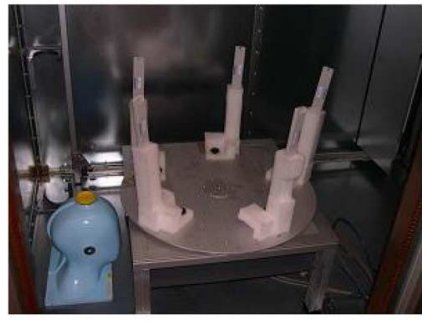

(b)
Fig. 5. Setup in reverberation chamber used for RFID tag population measurements. (a) Schematic figure showing possible positions on platform stirrer. (b) Photograph of setup.

Normally, when identifying tags in a population collisions between tags transmissions is not beneficial, the RFID reader can therefore create $2^{Q}$ (where $Q$ is a integer between 0 and 15) number of time slots, from which tags randomly pick one timeslot for their transmission, hence lowering the probability that many tags will transmit during the same timeslot. By setting the $Q$-value to zero, all tags in the readable zone will respond instantly and in the same timeslot, hence there will be a competition among the tags responses back to the reader, where none or only the strongest signal can be resolved.

Five tags were given unique 96-bit EPC values and placed at an equal distance from their neighbor tag, facing outwards and $\lambda / 2$ from any metal or lossy object; see Fig. 5 . The stirrers were moved continuously for $2 \mathrm{~min}$. The number of 96-bit EPC reads from each tag during the stirrer movement was recorded by the controlling software.

To verify the position independence, the above measurement procedure was repeated five times for each measurement the tag positions were changed, and repeated until all five tags had been on all five positions. The results showed that the number of read 96-bit EPCs is not a property of position in the chamber, but rather a property of the tag itself. The mean standard error in terms of percentage of total number of read EPCs over the five different positions was $\sigma_{\text {mean,all }}=2 \%$.

The effect of the transmitted power level was also studied, and the conclusion was that population measurements must be done at quite low transmit power from the reader. This is to ensure that tag sensitivity will be the dominant factor determining the number of successfully read EPCs during the fading. The effect of tag sensitivity differences will evidently be larger when the power level radiated from the reader is low.

Using this method, it would be possible to make fast determination of the quality of tags in a population. By introducing a reference tag with known sensitivity, it would be possible to rank the tags under test as better or worse than the reference tag.

\section{CONCLUSION}

We have made a first attempt to introduce UHF RFID tag sensitivity measurement methods in a reverberation chamber. We have shown that we can distinguish tags of different sensitivity with good accuracy and verified the result with traditional anechoic chamber measurements. We have also successfully found a way to determine tag sensitivity from single-power- level measurement by treating the tag as an ideal threshold receiver as defined in [9] and using the statistics of a Rayleigh fading environment.

We have also further elaborated how sensitivity measurements can be made useful for finding the better tags among a population of various tags.

\section{ACKNOWLEDGMENT}

The authors are thankful to M. Andersson, G. Ivansson (Learningwell.se), and L. Andersson (Swedish Transport Administration) for providing the RFID equipment. Special thanks is also given to S. Sjögren at Scirocco and to Siemens for the technical support, and to J. Yang for his help.

\section{REFERENCES}

[1] J. Landt, "The history of RFID," IEEE Potentials, vol. 24, no. 4, pp. 8-11, Oct.-Nov. 2005.

[2] A. Lazaro, D. Girbau, and D. Salinas, "Radio link budgets for UHF RFID on multipath environments," IEEE Trans. Antennas Propag., vol. 57, no. 4, pp. 1241-1251, Apr. 2009.

[3] P. V. Nikitin, K. V. S. Rao, R. Martinez, and S. F. Lam, "Sensitivity and impedance measurements of UHF RFID chips," IEEE Trans. Microw. Theory Tech., vol. 57, no. 5, pp. 1297-1302, May 2009.

[4] M. Ritamaki, A. Ruhanen, V. Kukko, J. Miettinen, and L. H. Turner, "Contactless radiation pattern measurement method for UHF RFID transponders," Electron. Lett., vol. 41, pp. 723-724, 2005.

[5] P.-S. Kildal and K. Rosengren, "Correlation and capacity of MIMO systems and mutual coupling, radiation efficiency, and diversity gain of their antennas: Simulations and measurements in a reverberation chamber," IEEE Commun. Mag., vol. 42, no. 12, pp. 104-112, Dec. 2004.

[6] K. Rosengren and P.-S. Kildal, "Study of distributions of modes and plane waves in reverberation chambers for the characterization of antennas in a multipath environment," Microw. Opt. Technol. Lett., vol. 30, pp. 386-91, 2001.

[7] K. Rosengren and P.-S. Kildal, "Radiation efficiency, correlation, diversity gain and capacity of a six-monopole antenna array for a MIMO system: Theory, simulation and measurement in reverberation chamber," Inst. Elect. Eng. Proc., Microw., Antennas Propag., vol. 152 , pp. 7-16, 2005.

[8] C. Orlenius, P.-S. Kildal, and G. Poilasne, "Measurements of total isotropic sensitivity and average fading sensitivity of CDMA phones in reverberation chamber," in Proc. IEEE Antennas Propag. Soc. Int. Symp., Jul. 3-8, 2005, vol. 1A, pp. 409-12.

[9] P.-S. Kildal, A. Hussain, X. Chen, C. Orlenius, A. Skårbratt, J. Åsberg, T. Svensson, and T. Eriksson, "Threshold receiver model for throughput of wireless devices with MIMO and frequency diversity measured in reverberation chamber," IEEE Antennas Propag. Wireless Lett., vol. 10, pp. 1201-1204, 2011.

[10] D. A. Hill, M. T. Ma, A. R. Ondrejka, B. F. Riddle, M. L. Crawford, and R. T. Johnk, "Aperture excitation of electrically large, lossy cavities," IEEE Trans. Electromagn. Compat., vol. 36, no. 3, pp. 169-178, Aug. 1994.

[11] J. G. Kostas and B. Boverie, "Statistical model for a mode-stirred chamber," IEEE Trans. Electromagn. Compat., vol. 33, no. 4, pp. 366-370, Nov. 1991.

[12] X. Chen, P.-S. Kildal, J. Carlsson, and J. Yang, "Comparison of ergodic capacities from wideband MIMO antenna measurements in reverberation chamber and anechoic chamber," IEEE Antennas Wireless Propag. Lett., vol. 10, pp. 446-449, 2011.

[13] X. Chen, P.-S. Kildal, C. Orlenius, and J. Carlsson, "Channel sounding of loaded reverberation chamber for over-the-air testing of wireless devices: Coherence bandwidth versus average mode bandwidth and delay spread," IEEE Antennas Wireless Propag. Lett., vol. 8, pp. 678-681, 2009.

[14] O. Delangre, P. De Doncker, M. Lienard, and P. Degauque, "Delay spread and coherence bandwidth in reverberation chamber," Electron. Lett., vol. 44, pp. 328-329, 2008

[15] E. Genender, C. L. Holloway, K. A. Remley, J. M. Ladbury, G. Koepke, and H. Garbe, "Simulating the multipath channel with a reverberation chamber: Application to bit error rate measurements," IEEE Trans. Electromagn. Compat., vol. 52, no. 4, pp. 766-777, Nov. 2010. 